\title{
Stability of the COVID-19 virus under wet, dry and acidic conditions
}

Zhi-ping Sun ${ }^{1 *}$, Xia Cai ${ }^{1^{*}}$, Chen-jian $\mathrm{Gu}^{2 *}$, Rong Zhang ${ }^{2 *}$, Wen-dong Han ${ }^{1}$, Yun Qian ${ }^{1}$, Yu-yan $\mathrm{Wang}^{2}$, Wei $\mathrm{Xu}^{2}$, Yang $\mathrm{Wu}^{2}$, Xunjia Cheng ${ }^{2}$, Zheng-hong Yuan ${ }^{2 * *}$, You-hua $\mathrm{Xie}^{2 * *}, \mathrm{Di} \mathrm{Qu}^{1,2^{* *}}$

${ }^{1}$ BSL-3 laboratory of Fudan University, School of Basic Medical Sciences, Shanghai Medical College, Fudan University

${ }^{2}$ Key Laboratory of Medical Molecular Virology (MOE/NHC/CAMS), Department of Medical Microbiology and Parasitology, School of Basic Medical Sciences, Shanghai Medical College, Fudan University

* Co-first author

${ }^{* *}$ Corresponding author

Di Qu:dqu@shmu.edu.cn

You-hua Xie: yhxie@fudan.edu.cn

Zheng-hong Yuan: zhyuan@shmu.edu.cn 
medRxiv preprint doi: https://doi.org/10.1101/2020.04.09.20058875; this version posted April 14, 2020. The copyright holder for this preprint

To the Editor,

An outbreak of pneumonia caused by a novel coronavirus was reported in December 2019 in Wuhan, Hubei Province of China ${ }^{[1-3]}$. The COVID-19 virus (SARSCOV-2) has spread to every continent and WHO declared COVID-19 as a pandemic ${ }^{[4]}$. In this study, the presence of CPE was used as the main index for the detection of the infectivity of the COVID-19 virus. When CPE was not observed, immunofluorescence of intracellular viral N protein and RT-PCR of viral RNA in the cell culture supernatant were used for the detection of viral infection. The strain nCoV-SH01 (GenBank accession no. MT121215) used in this study was isolated and plaque -purified from the nasal-pharyngeal swab of a clinically confirmed COVID-19 patient in Shanghai ${ }^{[5]}$.

Using the strain nCoV-SH01 and these criteria, we first investigated the infectivity of the COVID-19 virus. We found that as the virus titer decreases $(2000,1000,500$, 250, 100, 50, 10, 5, and $1 \mathrm{PFU})$, the time for CPE to appear is delayed. One PFU is able to cause infection of Vero-E6 cells, resulting in obvious CPE at 72 hours, whereas higher titer of the virus induced CPE at 24 hours post inoculation (Table 1). It indicates that the COVID-19 virus is highly infectious, and underscores the challenge to control the spread of the COVID-19 virus.

We then studied the stability of the COVID-19 virus in the wet (in $100 \mathrm{uL}$ culture medium) and dry (10 uL supernatant on filter paper) environments at room temperature $\left(22^{\circ} \mathrm{C}\right)$ for $1,2,3,4,5,6,7$ days respectively. Our results show that the COVID-19 virus can survive for 3 days in the wet or dry environment investigated in this study. Although the virus maintained its infectivity within 3 days in the dry condition, CPE appeared later than that kept in the wet environment, indicating that the dry environment may be less favorable for the survival of the COVID-19 virus (Table $2 \&$ Table 3 ). However, when the virus had been kept in the wet or dry condition for more than 4 days, no CPE was observed (Table $2 \&$ Table 3), which was confirmed by immune florescence staining (data not shown) with the antibody against viral $\mathrm{N}$ protein as well as qRT-PCR.

We further investigated the stability of the COVID-19 virus at $\mathrm{pH} 2.2$ condition. It shows that the COVID-19 virus has a certain degree of tolerance to acidic environment. In the present study, when $1.2 \times 10^{3} \mathrm{PFU}$ of the COVID-19 virus were treated with acidic saline of $\mathrm{pH} 2.2$ for 30 or 60 minutes, it still resulted in CPE in the cells, whereas $1.0 \times 10^{3} \mathrm{PFU}$ of the COVID-19 virus treated with $\mathrm{pH} 2.2$ saline for 30 or 60 minute, no CPE were observed (Table 4). It suggests under the acidic condition the COVID-19 
medRxiv preprint doi: https://doi.org/10.1101/2020.04.09.20058875; this version posted April 14, 2020. The copyright holder for this preprint (which was not certified by peer review) is the author/funder, who has granted medRxiv a license to display the preprint in perpetuity.

It is made available under a CC-BY-NC-ND 4.0 International license .

virus at a relatively high titer can survive under acidic condition for at least 1 hour.

In conclusion, our findings show that the COVID-19 virus is highly infectious that one PFU can results in cell infection in our in vitro system, and can survive for 3 days in the wet or dry environment. In addition, we for the first time demonstrated that the COVID-19 virus at relative high concentrations can survive under acidic condition that minic the gastric environment. The study would also provide guidance on taking appropriate measures to control the spread of the COVID-19 virus and improve laboratory safety.

The study was supported by the National Science and Technology Major Project (NSTMP) for the Prevention and Treatment of Infectious Diseases (2018ZX10734401, 2018ZX10301208), NSTMP for the Development of Novel Drugs (2019ZX09721001), and Project of Novel Coronavirus Research of Fudan University.

\section{Reference}

1. Wang, C., et al., A novel coronavirus outbreak of global health concern. Lancet, 2020. 395(10223): p. 470-473.

2. Qun Li, X.G., Peng Wu, Xiaoye Wang, Lei Zhou, Yeqing Tong, Ruiqi Ren, Kathy S.M. Leung, Eric H.Y. Lau, Jessica Y. Wong, Xuesen Xing, Nijuan Xiang, Yang Wu, Chao Li, Qi Chen, Dan Li, Tian Liu, Jing Zhao, Man Liu, Wenxiao Tu, Chuding Chen, Lianmei Jin, Rui Yang, Qi Wang, Suhua Zhou, Rui Wang, Hui Liu, Yinbo Luo, Yuan Liu, Ge Shao, Huan Li, Zhongfa Tao, Yang Yang, Zhiqiang Deng, Boxi Liu, Zhitao Ma, Yanping Zhang, Guoqing Shi, Tommy T.Y. Lam, Joseph T. Wu, George F. Gao, D.Phil., Benjamin J. Cowling, Bo Yang, Gabriel M. Leung, Zijian Feng, Early Transmission Dynamics in Wuhan, China, of Novel Coronavirus-Infected Pneumonia. The New England Journal of Medicine, 2020(382): p. 1199.

3. The 2019-nCoV Outbreak Joint Field Epidemiology Investigation Team, Q.L., Notes from the Field: An Outbreak of NCIP (2019-nCoV) Infection in China - Wuhan, Hubei Province, 20192020. China CDC Weekly, 2020. 2(5): p. 79-80.

4. Organization, W.H. There is a current outbreak of Coronavirus (COVID-19) disease. 2020; Available from: https://www.who.int/health-topics/coronavirus\#tab=tab_1.

5. Zhang Rong, Y.Z., Wang Yuyan, Teng Zheng, Xu Wei, Song Wuhui, Cai Xia, Sun Zhiping, Gu Chenjian, Zhou Yanqiu, Chen Hongyou, Ye Rong, Han Wendong, Zhu Yunkai, Feng Fanghao, Li Chongshan, Zhang Xi, Qu Di, Fu Chen, Xie Youhua, Yuan Zhenghong, Isolation of a 2019 novel coronavirus strain from a coronavirus disease 19 patient in Shanghai. Journa of Microbes and Infections, 2020. 15(1): p. 15-20. 
Table 1. The cytopathic effect of Vero-E6 cell infected by different PFU of the COVID-19 virus

\begin{tabular}{|c|c|c|c|c|c|c|c|c|c|c|c|c|}
\hline \multirow{2}{*}{$\begin{array}{c}\text { Time } \\
\text { (d.p.i.)\# }\end{array}$} & \multirow{2}{*}{$\begin{array}{c}\text { Cell } \\
\text { control }\end{array}$} & \multicolumn{11}{|c|}{ Viral inoculation $\left(\times 10^{2} \mathrm{PFU}\right)^{*}$} \\
\hline & & 20 & 10 & 5 & 2.5 & 1 & 0.5 & 0.1 & 0.05 & & $0.01 * *$ & \\
\hline 1 & - & ++++ & ++++ & ++ & + & - & - & - & - & - & - & - \\
\hline 2 & - & I & ++++ & ++++ & ++++ & ++++ & ++++ & ++++ & ++++ & ++++ & - & - \\
\hline 3 & - & 1 & / & / & / & / & / & ++++ & ++++ & ++++ & ++++ & ++++ \\
\hline
\end{tabular}

\#, d.p.i. days post-inoculation. CPE of Vero E6 cells was checked under microscope. Degree of CPE, “++++”, $>75 \%$ of cells; “+++”, $50 \% \sim 75 \%$; “++”, $25 \%$ $50 \%$; “+”, $0 \sim 25 \%$

* The experiments were carried out in triplicate wells for each dilution. The cell control was without the virus. The cytopathic effects were observed under a microscope daily for 3 days.

** In the triplicate wells inoculated with $0.01 \times 10^{2} \mathrm{PFU}, \mathrm{CPE}$ appeared at different time points in individual wells and was separately presented. 
Table 2. Stability of the COVID-19 virus under wet condition

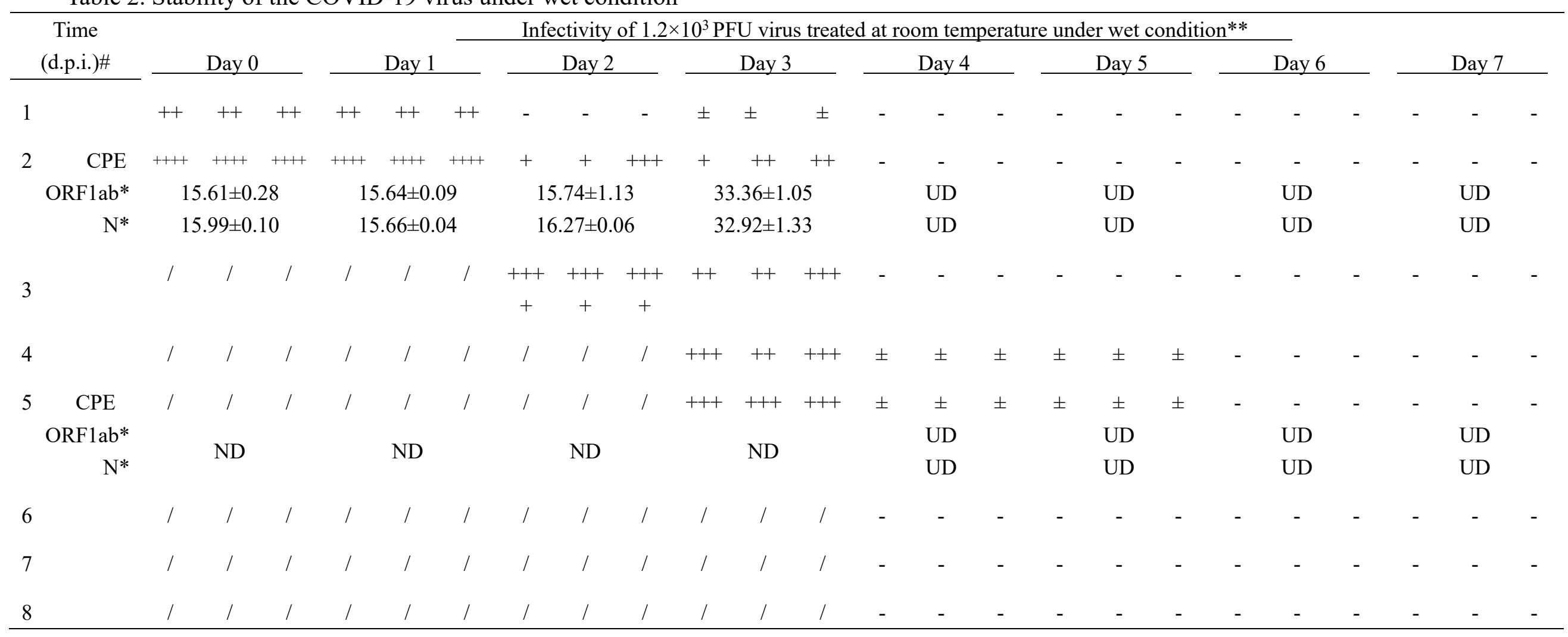

\#, d.p.i. days post-inoculation. CPE of Vero E6 cells was checked under microscope. Degree of CPE, “++++”, $>75 \%$ of cells; “+++", $50 \% \sim 75 \%$; “++”, $25 \% \sim 50 \%$; “+”, 0 25\%; “土”, not clear-cut; “-”, no CPE.

* The Viral RNA in the supernatant after 2-day and 5-day incubation was extracted. Quantitative RT-PCR was performed using the primers and probes for viral ORF1 ab and N (listed in Table 1). Ct values were presented as Mean \pm SD. Cutoff value: Ct value > 38. UD: under detectable level. ND: Not determined.

** The experiments were carried out in triplicate wells for each dilution. The cytopathic effects were observed under a microscope daily for 8 days. 
Table 3. Stability of the COVID-19 virus under dry condition.

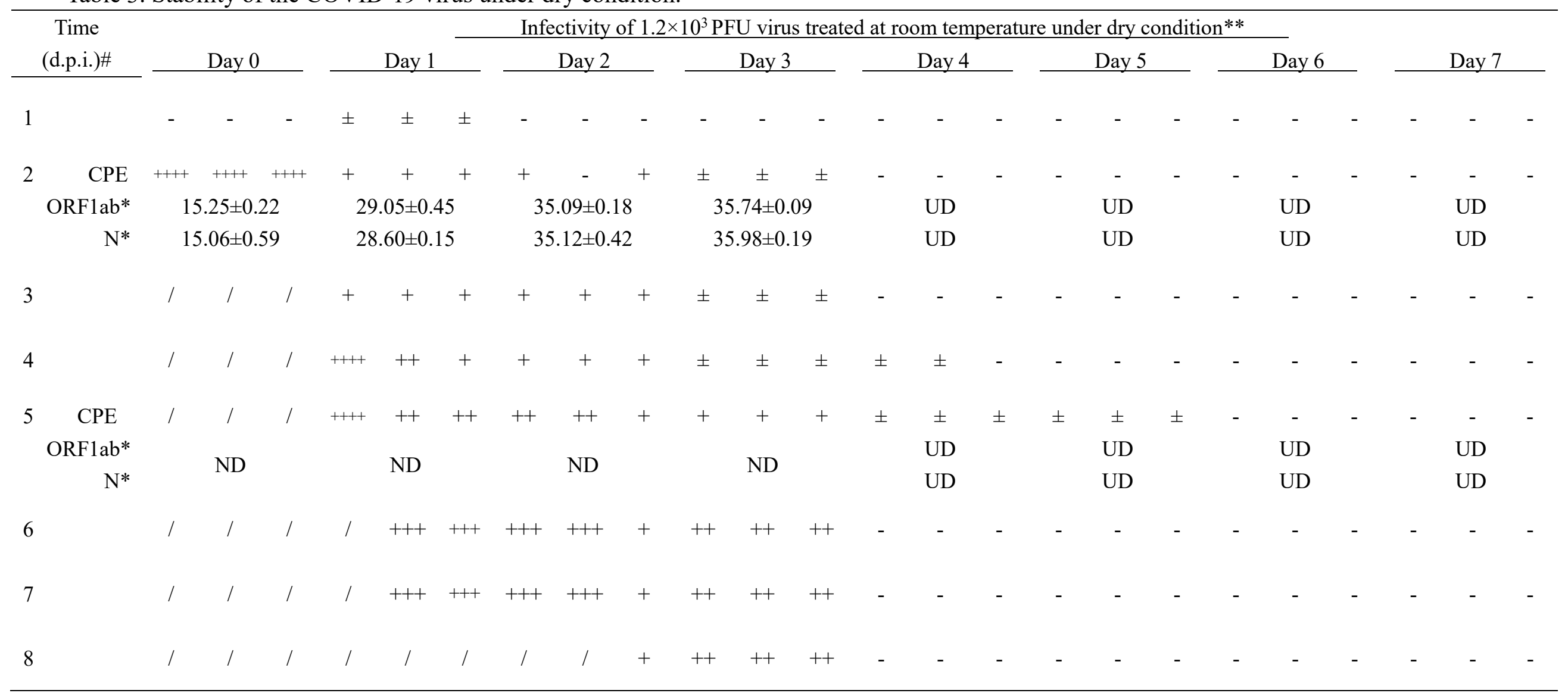

\#, d.p.i. days post-inoculation. CPE of Vero E6 cells was checked under microscope. Degree of CPE, “++++”, >75\% of cells; “+++”, 50\% 75\%; “++”, $25 \% \sim 50 \%$; “+”, 0 25\%; “土”, not clear-cut; “-”, no CPE.

* The Viral RNA in the supernatant after 2-day and 5-day incubation was extracted. Quantitative RT-PCR was performed using the primers and probes for viral ORF1 ab and N (listed in Table 1). Ct values were presented as Mean \pm SD. Cutoff value: Ct value > 38. UD: under detectable level. ND: Not determined.

** The experiments were carried out in triplicate wells for each dilution. The cytopathic effects were observed under a microscope daily for 8 days. 
Table 4. Stability of the COVID-19 virus under acidic condition

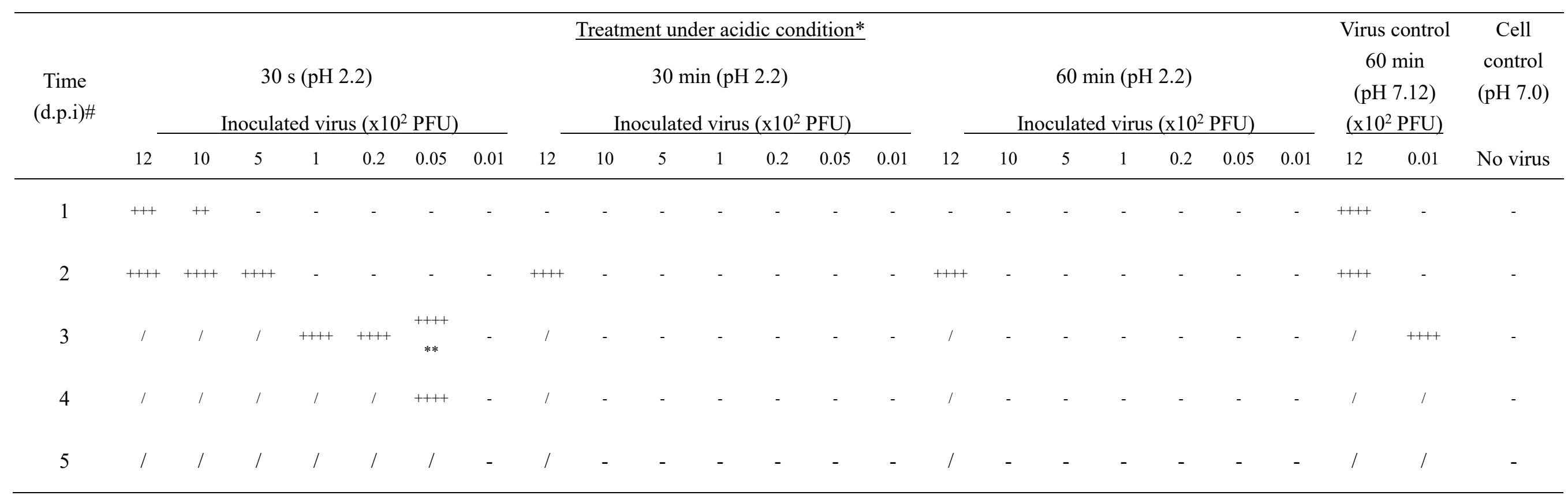

\#, d.p.i. days post-inoculation. CPE of Vero E6 cells was checked under microscope. Degree of CPE, “++++”, >75\% of cells; “+++", 50\% 75\%; “++”, 25\% 50\%; “+”, 0 25\%; “"”, not clear-cut; “-”, no CPE.

* The experiments were carried out in triplicate wells for each dilution. The virus control was treated with physiological saline (final $\mathrm{pH}=7.12)$ for $60 \mathrm{~min}$. Physiological saline $(\mathrm{pH}=7.0)$ was used as a blank control. The cytopathic effects were observed under a microscope daily for 5 days.

** The $0.05 \times 10^{2} \mathrm{PFU}$ group ( 30 seconds) showed CPE (++++) in 1 well at $72 \mathrm{hrs}$, and all become ++++ at $96 \mathrm{hrs}$. 